\title{
Nicotine-selective radiation-induced poly(acrylamide/maleic acid) hydrogels
}

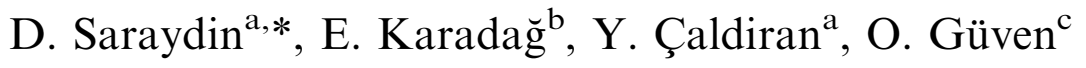 \\ ${ }^{a}$ Department of Chemistry, Cumhuriyet University, 58140 Sivas, Turkey \\ ${ }^{\mathrm{b}}$ Department of Chemistry, Adnan Menderes University, 09010 Aydin, Turkey \\ ${ }^{\mathrm{c}}$ Department of Chemistry, Hacettepe University, 06532 Beytepe/Ankara, Turkey
}

Received 13 March 2000; accepted 19 August 2000

\begin{abstract}
Nicotine-selective poly(acrylamide/maleic acid) (AAm/MA) hydrogels prepared by $\gamma$-irradiation were used in experiments on swelling, diffusion, and interactions of the pharmaceuticals nicotine, nicotinic acid, nicotinamide, and nikethamide. For AAm/MA hydrogel containing $60 \mathrm{mg}$ maleic acid and irradiated at $5.2 \mathrm{kGy}$, the studies indicated that swelling increased in the following order; nicotine $>$ nicotinamide $>$ nikethamide $>$ nicotinic acid $>$ water. Diffusions of water and the pharmaceuticals within the hydrogels were found to be non-Fickian in character. AAm/MA hydrogel sorbed only nicotine and did not sorb nicotinamide, nikethamide and nicotinic acid in the binding experiments. S-type adsorption in Giles's classification system was observed. Some binding and thermodynamic parameters for AAm/MA hydrogel-nicotine system were calculated using the Scatchard method. The values of adsorption heat and free energy of this system were found to be negative whereas adsorption entropy was found to be positive. (C) 2001 Elsevier Science Ltd. All rights reserved.
\end{abstract}

Keywords: Hydrogel; Poly(acrylamide/maleic acid); Nicotine; Sorption; Interaction

\section{Introduction}

Hydrophilic polymers when cross-linked chemically or physically swell but do not dissolve in water. They are termed hydrogels when the amount of water retained is between 20 and $100 \%$ of the total mass (Güven et al., 1999). The interactions between polymers and cosolutes are both biochemically significant and scientifically interesting, and they are also technologically important in several respects. There are several techniques for the immobilization of biomolecules on and within hydrogels, including physical entrapment, electrostatic attraction, physical adsorption with or without crosslinking, and chemical bonding. In particular, physical adsorption via secondary molecular forces is an

\footnotetext{
*Corresponding author. Fax: +90-346-219-1186.

E-mail address: saraydin@cumhuriyet.edu.tr (D. Saraydin).
}

important way to immobilize a biological species on a hydrogel (Kaetsu, 1996; Kost et al., 1987; Rosiak et al., 1983).

Environmental sensitivity or control of swelling ability of poly-electrolyte hydrogels under a variety of external conditions make them ideal adsorbents for the removal, adsorption and enrichment of some watersoluble agents such as biomolecules, proteins, metallic impurities, dyes, etc. The hydrogels based on polyelectrolyte structures and synthesized via irradiation of aqueous solutions of acrylamide with small quantities of maleic or itaconic acids are regarded as system with potential immobilization, chelating and adsorptive properties for various bioapplications (Güven et al., 1999).

In our previous articles, acrylamide-based hydrogels have been studied in adsorption of protein (Saraydin et al., 1994; Karadağ et al., 1994; Akkaş et al., 1999; 
Tümtürk et al., 1999) and biocompatibility of human sera (Saraydin et al., 1995a; Karadağ et al., 1996a). In addition, the binding of the nicotine to acrylamide/ itaconic acid hydrogels has been studied (Karadağ et al., 1997). In this study, the interactions between nicotine (1-methyl-2-(3-pyridyl) pyrrolidine), nicotinic acid (niacin, 3-pyridine carboxylic acid, Vitamin PP), nicotinamide (niacinamid, 3-pyridinecarboxamide, Vitamin $\mathrm{B}_{3}$, Vitamin PP), and nikethamide (nicamide, $N, N$-diethyl nicotinamide) with novel hydrogels prepared with acrylamide and maleic acid were investigated.

\section{Experimental}

The two monomers used in this study, namely, acrylamide (AAm) and maleic acid (MA) were obtained from BDH (Poole, UK), and nicotine, nicotinic acid, nicotinamide and nikethamide from Sigma (St. Louis, MO, USA). A suitable mass of MA and irradiation doses for AAm and MA hydrogels were selected by taking previous experiments into consideration (Saraydin et al., 1995b).

Aqueous solutions of monomers of $1 \mathrm{~g}$ AAm and 20, $30,40,50$ and $60 \mathrm{mg}$ MA were prepared in $1 \mathrm{ml}$ of pure water. Monomer solutions thus prepared were placed in the PVC straws of $3 \mathrm{~mm}$ diameter and irradiated to 2.60 , $3.73,4.65,5.20$ and $5.71 \mathrm{kGy}$ in air at ambient temperature in a $\gamma$-cell 220-type $\gamma$-irradiator at a fixed dose rate of $0.72 \mathrm{kGy} \mathrm{h}^{-1}$. Hydrogels obtained in long cylindrical shapes were cut into pieces of $4-5 \mathrm{~mm}$ long, dried first in air and then in vacuum.

AAm hydrogel and AAm/MA hydrogel containing $60 \mathrm{mg} \mathrm{MA}$ and irradiated to $5.2 \mathrm{kGy}$ were swollen in distilled water and in $100 \mathrm{mg}^{-1}$ nicotine, nicotinic acid, nicotinamide and nikethamide solutions at $25^{\circ} \mathrm{C}$ to determine the parameters of swelling and diffusion. Swollen gels removed from the water bath at regular intervals were dried superficially with filter paper, weighed, and placed in the same bath. The radii of cylindrical swollen gels were measured by a micrometer.

Aqueous solutions of nicotine were prepared in the concentration range of $20-100 \mathrm{mg}^{-1}$. AAm/MA hydrogels containing $60 \mathrm{mg} \mathrm{MA}$ and irradiated to $5.2 \mathrm{kGy}$ weighing $0.1 \mathrm{~g}$ were transferred into $50 \mathrm{ml}$ of aqueous solutions of nicotine and allowed to equilibrate for $24 \mathrm{~h}$ at $25^{\circ} \mathrm{C}$. These solutions were separated by decantation from the hydrogels. Spectrophotometric measurements of these nicotine solutions were carried out using a Shimadzu A160 model UV-VIS double beam spectrophotometer at ambient temperature. The absorbance of these solutions was recorded at a wavelength of $261 \mathrm{~nm}$. Distilled water was chosen as a reference.

The influences of MA content and irradiation dose were investigated for binding of nicotine to AAm/MA hydrogels. Hydrogel samples weighing $0.1 \mathrm{~g}$ and pre- pared with different MA content and irradiation doses were added to $50 \mathrm{ml}$ of $50 \mathrm{mgl}^{-1}$ nicotine. The samples were left in the solution for one day at $25^{\circ} \mathrm{C}$ and the solutions were measured spectrophotometrically.

\section{Results and discussion}

\subsection{Swelling and diffusion}

The swelling of AAm/MA containing $60 \mathrm{mg}$ MA irradiated at $5.2 \mathrm{kGy}$ in water and the pharmaceuticals was calculated from the following relation:

$S=\frac{m_{\mathrm{t}}-m_{0}}{m_{0}}$

where $m_{\mathrm{t}}$ is the mass of the swollen gel at time $t$ and $m_{0}$ is the mass of the dry gel. Swelling curves of AAm/MA in distilled water and the solutions of the pharmaceuticals are shown in Fig. 1.

The equilibrium swelling of hydrogels is between 17 and 22 in the aqueous solutions of the pharmaceuticals, but 13 in distilled water. The pharmaceuticals contain unpaired electrons in $\mathrm{N}$ and $\mathrm{O}$ (Scheme 1). These atoms behave like a hydrophilic group and form hydrogen bonds with water. Thus, the swelling of AAm/MA hydrogels changes as the pharmaceuticals bring water into the gels. On the other hand, the polar pyrrolidine ring (due to second dissociation) in the nicotine molecule can cause a larger degree of swelling. Nicotinamide and nikethamide molecules contain amide groups which are similar to (AAm) molecules. Thus, amide groups in nicotinamide, nikethamide and acrylamide can interact, which results in more pronounced swelling of the hydrogel in these solutions than that is encountered in the solution of nicotinic acid. On the other hand, there will be anionic repulsion between carboxylic groups of

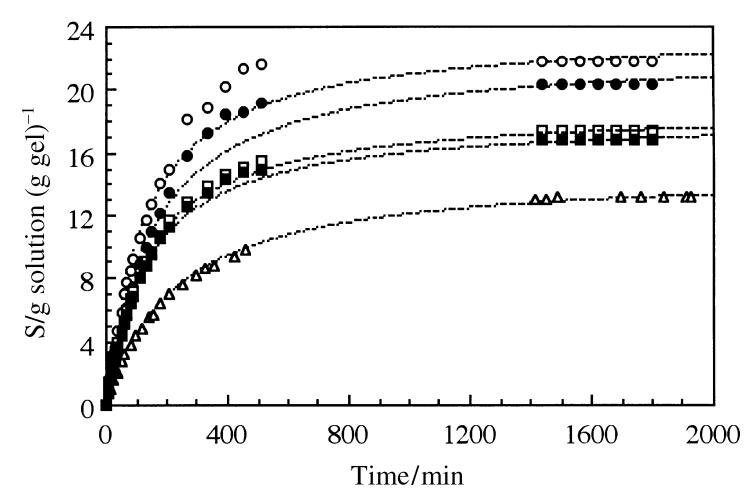

Fig. 1. The swelling curves of AAm/MA hydrogel in the solutions. ( $\bigcirc)$ nicotine, (○) nicotinamide, ( $\square$ ) nikethamide, (ם) nicotinic acid, $(\triangle)$ water, $(\ldots \ldots)$ theoretical swelling curves from Eq. (2). 
<smiles>CN1CCCC1c1cccnc1</smiles><smiles>NC(=O)c1cccnc1</smiles>

nicotinamide<smiles>CCN(CC)C(=O)c1cccnc1</smiles>

nikethamide<smiles>O=C(O)c1cccnc1</smiles>

nicotinic acid

Scheme 1.

nicotinic acid and maleic acid in the hydrogel, so, the swelling of the hydrogels is decreased to 17 . At the end of these evaluations, the superswelling polyelectrolytic AAm/MA hydrogel in the aqueous solutions are swollen by the following order: nicotine $>$ nicotinamide $>$ nikethamide $>$ nicotinic acid $>$ water.

For extensive swelling of polymers, the following relation can be written (Peniche et al., 1997) as

$\frac{t}{S}=A+B t$,

where $S$ is the degree of the swelling at time $t$, $B=1 / S_{\max }$ is the inverse of the maximum or equilibrium swelling, $A=1 /(\mathrm{d} S / \mathrm{d} t)_{0}$, is the reciprocal of the initial swelling rate $\left(r_{0}\right)$ of the gel. The relation represents second-order kinetics.

Fig. 2 shows the linear regression of the swelling curves obtained by means of Eq. (2). The initial swelling rate and the values of maximum swelling of the hydrogels were calculated from the slope of an intersection of the lines and, are presented in Table 1. The values of maximum swelling of the hydrogelspharmaceutical systems suggest similar swelling behavior (Table 1).

Analysis of the mechanisms of water diffusion in swellable polymeric systems has received considerable attention in recent years because of the important applications of swellable polymers in the biomedical, pharmaceutical, environmental, and agricultural fields. The following equation was used to determine the nature of diffusion of water, nicotine, nicotinic acid, nicotinamide, and nikethamide into hydrogels (Peppas and Franson, 1983; Ritger and Peppas, 1987):

$F=k t^{n}$,

where $F$ denote the fraction of the solvent which diffused into the gel at time $t . k$ is a constant incorporating characteristic of the polymeric network system and the penetrant and $n$ is the diffusional exponent, which is indicative of the transport mechanism. Eq. (3) is valid for the first $60 \%$ of the normalized solvent uptake. Fickian diffusion and Case II transport are defined by $n$ equal to $\frac{1}{2}$ and $n$ equal to 1 , respectively. Anomalous transport behavior (non-Fickian diffusion) is defined by values of $n$ between $\frac{1}{2}$ and 1 (Ritger and Peppas, 1987). This equation was applied to the initial stages of swelling, and plots of $\ln F$ versus $\ln t$ shown in Fig. 3. The exponents of $n$ and $k$ values were calculated from

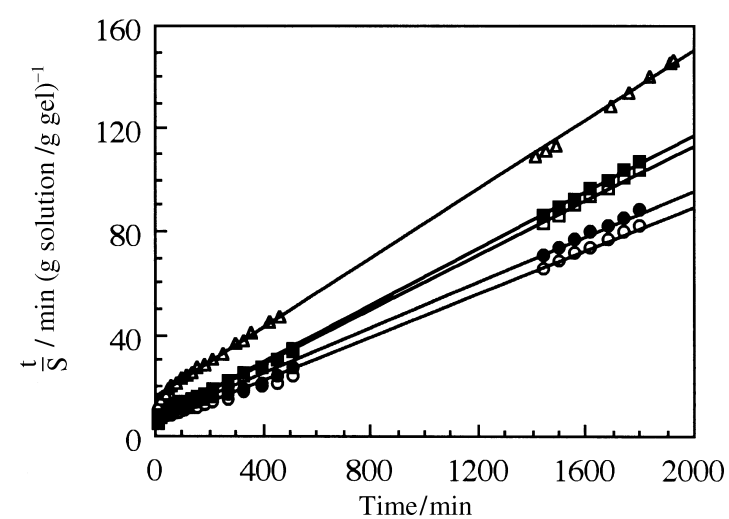

Fig. 2. The curves of swelling kinetics of AAm/MA hydrogel in the solutions. ( $\bigcirc)$ nicotine, $(\bigcirc)$ nicotinamide, $(\square)$ nikethamide, $(\boldsymbol{\square})$ nicotinic acid, $(\triangle)$ water.

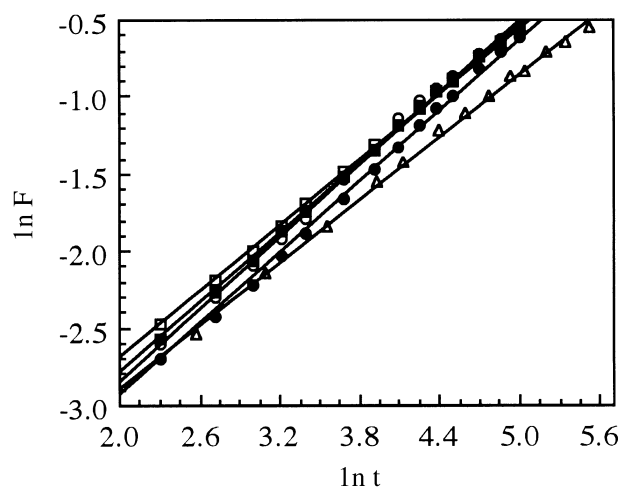

Fig. 3. The $\ln F-\ln t$ curves for AAm/MA hydrogel in the solutions. $(\bigcirc)$ nicotine, $(\bigcirc)$ nicotinamide, $(\square)$ nikethamide, (ם) nicotinic acid, $(\triangle)$ water.

the slope and intercept of the lines, respectively, and are presented in Table 1.

$n$ was found between 0.50 and 1 (Table 1); and hence, the diffusion of the pharmaceuticals into AAm/MA hydrogels was taken to be of non-Fickian character. This is generally explained as being a consequence of the slow relaxation rate of the hydrogel (Ritger and Peppas, 1987).

The study of diffusion phenomena in hydrogels and water is of value in that it clarifies polymer behavior. For hydrogel characterization, the diffusion coefficient 
Table 1

The parameters of swelling and diffusion of AAm/MA hydrogel

\begin{tabular}{llllllr}
\hline Solution & $S_{\text {exp }}$ & $S_{\max }$ & $r_{0} \times 10^{2}$ & $k \times 10^{2}$ & $n$ & $D \times 10^{7}\left(\mathrm{~cm}^{2} \mathrm{~s}^{-1}\right)$ \\
\hline Water & 13 & 15 & 6.52 & 1.43 & 0.68 & 7.25 \\
Nicotinic acid & 17 & 18 & 13.23 & 1.38 & 0.75 & 11.19 \\
Nikethamide & 17 & 19 & 14.07 & 1.67 & 0.71 & 11.32 \\
Nicotinamide & 20 & 22 & 14.45 & 1.19 & 0.76 & 13.58 \\
Nicotine & 22 & 24 & 18.55 & 1.22 & 0.78 & 14.01 \\
\hline
\end{tabular}

$D$ can be calculated by various methods. The short-time approximation method was used for calculation of diffusion coefficients of AAm/MA hydrogels (Peppas and Franson, 1983). The short-time approximation is valid for the first $60 \%$ of the swelling. The diffusion coefficients $D$ of the cylindrical AAm/MA hydrogels were calculated from the following relations:

$F=4\left[\frac{D t}{\pi l^{2}}\right]^{1 / 2}-\pi\left[\frac{D t}{\pi l^{2}}\right]-\frac{\pi}{3}\left[\frac{D t}{\pi l^{2}}\right]^{3 / 2}$,

where $D$ in $\mathrm{cm}^{2} \mathrm{~s}^{-1}, t$ in seconds and $l$ is the radius of cylindrical polymer sample. A graphical comparison of Eqs. (3) and (4) shows the semi-empirical equation. (3) with $n=0.5$ and $k=4\left(D / \pi r^{2}\right)^{1 / 2}$. For the hydrogels, $F$ versus $t^{1 / 2}$ plots are plotted and, are shown in Fig. 4 . The values of the diffusion coefficients of AAm/MA hydrogels are listed in Table 1. The values of the diffusion coefficient varied from $7.25 \times 10^{-7}$ to $14.01 \times$ $10^{-7} \mathrm{~cm}^{2} \mathrm{~s}^{-1}$. This result is parallel to the swelling result of the hydrogels.

\subsection{Interactions of the pharmaceuticals with $A A m / M A$ hydrogel}

To observe binding of nicotine and its pharmaceutical derivatives to AAm and AAm/MA hydrogels were placed in aqueous solutions of nicotine and its pharmaceutical derivatives nicotinic acid, nicotinamide and nikethamide, and allowed to equilibrate for one day. At the end of this time, a significant difference was measured between initial and final concentration of the nicotine solution containing AAm/MA hydrogel, while no significant differences were measured between initial and final concentration of the other pharmaceutical solutions containing AAm and AAm/MA hydrogels. So, AAm hydrogel did not sorb nicotine or its pharmaceutical derivatives from the solutions, and AAm/MA hydrogel sorbed only nicotine. Nicotine was therefore selected for binding studies to radiation-induced AAm/ MA hydrogels.

AAm is a non-ionic polymer, and thus acrylamide hydrogel generally does not interact with many small molecules. By the addition of MA to (AAm, AAm) copolymer contains ionizable groups. These groups can interact with the small molecules and these molecules

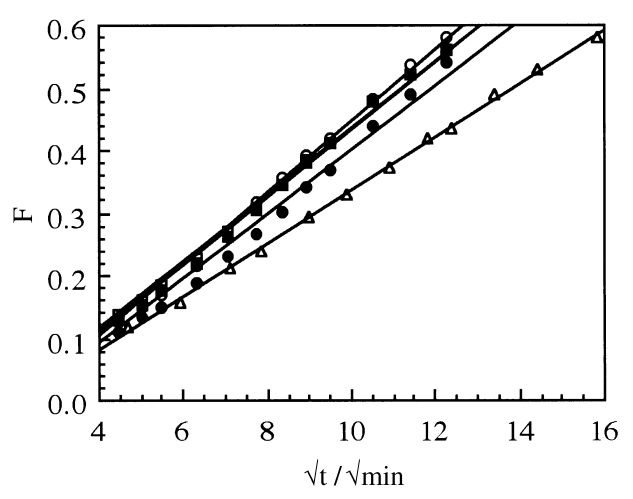

Fig. 4. The $F-\sqrt{t}$ curves for AAm/MA hydrogel in the solutions. ( $\bigcirc)$ nicotine, $(\bigcirc)$ nicotinamide, $(\square)$ nikethamide, (ם) nicotinic acid, $(\triangle)$ water.

bind to acrylamide copolymers with non-covalent or covalent bonds (Saraydin et al., 1995c; Saraydin et al., 1996a, b; Karadağ et al., 1995; Karadağ et al., 1996b, c). In the nicotine-AAm/MA hydrogel system, nicotine has a weak basic character, and the polar pyrrolidine ring in nicotine molecule, when nicotine has a positive charge, can interact by electrostatic forces with the carboxyl groups of maleic acid in the hydrogel (Scheme 2). Amide groups of nikethamide and nicotinamide cannot interact with maleic acid, because amide groups do not have ionic or polar character. On the other hand, there will be anionic repulsion between carboxyl groups of nicotinic acid and MA in the hydrogel, and therefore nicotinic acid does not bind to the AAm/MA hydrogel. According to these results, it could be concluded that the pyrrolidine- $N$ presumably plays more important role than the pyridine- $N$. The difference between the pyrrolidine- $N$ and pyridine- $N$ is their basicity. Therefore, the basicity of the functional group may be an important factor for binding to acrylamide hydrogel containing protic moieties (Matsui et al., 1996).

On the other hand, for a substrate molecule to get bounded to AAm/MA hydrogel, it has to penetrate first the outside aqueous layer and then with the specifically bound water molecules for the sites. Not only that, the structure of crosslinked polymer in the three-dimensional matrix would also change with changes in the water structure. Hence, any change in the amount of 


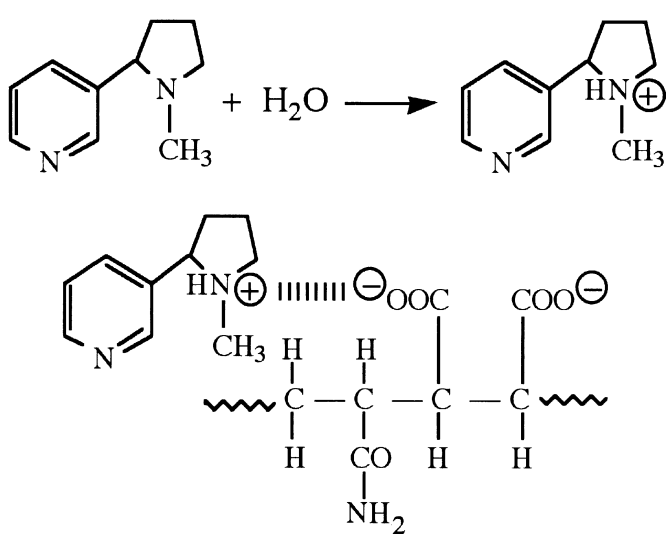

Scheme 2.

water sorbed could affect the binding sites and hence alter the type of interacting forces.

\subsection{Adsorption of nicotine onto AAm/MA hydrogel}

In a batch adsorption system at equilibrium, total cosolute concentration $\left(C_{\mathrm{I}}, \mathrm{moll}^{-1}\right)$ is

$C_{\mathrm{I}}=C_{\mathrm{B}}+C$,

where $C_{\mathrm{B}}$ is the equilibrium concentration of the cosolute on the adsorbent in mol per liter (bound cosolute concentration) and $C$ is the equilibrium concentrations of the cosolute in the solution in $\mathrm{mol} \mathrm{l}^{-1}$ (free cosolute concentration).The amount bound can therefore be conveniently expressed as the binding ratio, $r$, defined by

$r=C_{\mathrm{B}} / P$.

Thus, with $C_{\mathrm{B}}$ in mol $1^{-1}$ and $P$ in base mol (moles of monomer units, $P=m_{\mathrm{p}} / M$, where $m_{\mathrm{p}}$ is the mass of the polymer in $\mathrm{g}$ and $M$ is the molecular mass of repeating units of polymer in $\mathrm{g} \mathrm{mol}^{-1}$ ) per liter, $r$ then represents the average number of molecules of solute bound to each monomer unit at that free solute concentration. The binding data were interpreted on the basis of the uniform site-binding model (u.s.b.), which in statistical-thermodynamic terms corresponds to the formation of an ideal localised one-dimensional monolayer of cosolute on the polymer chains (Molyneux and Vekavakayanondha, 1986). This leads to the linear form of binding isotherm, which applies to many aqueous polymer/cosolute binding system (Molyneux, 1984)

$r=\frac{n K C}{1+K C}$,

where $K$ is the binding constant, that is, the equilibrium constant for the attachment of a molecule of nicotine- $N$ onto a site Si by a specific combination of non-covalent

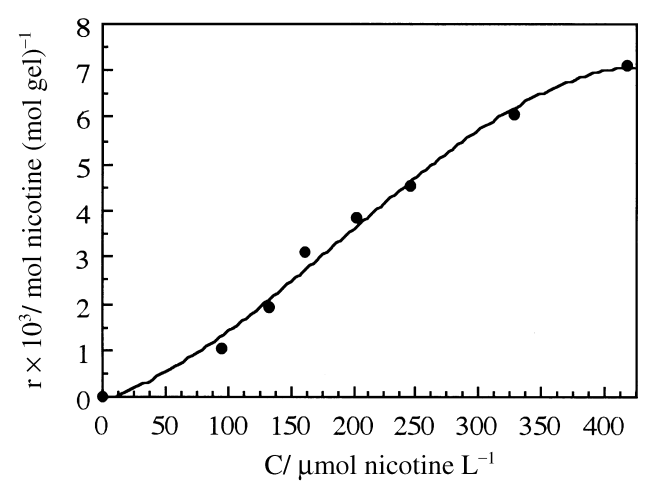

Fig. 5. The binding isotherm of nicotine - AAm/MA hydrogel binding system.

forces:

$\mathrm{N}+\mathrm{Si}=\mathrm{N} \sim \mathrm{Si}$

and $n$ is the site density (i.e. the limiting value of $r$ for "monolayer" coverage, which is thus a measure of the density of the sites $\mathrm{S}$ along the polymer chain). The reciprocal of $n$ is the site-size, $u$, which may be taken to represent either average number of monomer units occupied by the bound cosolute molecule, or more generally the average spacing of cosolute molecules when the chain saturated. The initial binding constant, $K_{i}$ is the initial slope of the binding isotherm, and thus represents the average binding strength of a cosolute molecule by a single-monomer unit on an occupied chain. In the u.s.b. model, it is equal to the product $K n$.

A plot of binding isotherm of nicotine is shown in Fig. 5. The binding of nicotine to AAm/MA hydrogel corresponds to S-type (sigmoidal) adsorption isotherms in the Giles classification system for adsorption of a cosolute from its solution (Fig. 5). In the $\mathrm{S}$ curves of the Giles classification system, the initial direction of the curvature shows that the adsorption is easier as the concentration rises. In practice, the $\mathrm{S}$ curve usually appear when three conditions are fulfilled: the solute molecule (a) is monofunctional, (b) has moderate intermolecular attraction, causing it to pack vertically in regular array in the adsorbed layer, and (c) meets strong competition, for substrate sites, from molecules of the solvent or of another absorbed species (Giles et al., 1960, 1974).

The weakness of the adsorbent-adsorbate forces will cause the uptake at low concentrations to be small, but once a molecule has become adsorbed, the adsorbateadsorbate forces will promote the adsorption of further molecules - a cooperative process - so that the isotherm will become convex to the concentration axis. S-type isotherms may originate through the adsorption 
of either non-polar molecules or polar molecules, always provided that the adsorbent-adsorbate force is relatively weak. A polar adsorbate of particular interest in this context is water, because the dispersion contribution to its overall interaction energy is unusually small compared with the polar contribution. Not surprisingly, water provided many examples of S-type isotherm. Once, the hydrogel is covered with a layer of adsorbed water; however, the adsorbent-adsorbate interaction would be virtually reduced to the weak dispersion energy of water with dyes, so that a S-type isotherm should result. In system that gives rise to an S-type isotherm, however, the multilayer is being built up on some parts of the surface whilst the monolayer is still incomplete on other parts (Giles et al., 1960, 1974).

The sorption mechanism, as implied by the S-type isotherm, is the attachment of the nicotine to AAm/MA hydrogel in vertical orientation (i.e. the nicotine molecule perpendicular to the plane of the hydrogel surface in which the binding site is present) through a monofunctional group and the stabilization of the same by intermolecular attraction existing between the sorbed molecules. On the examining the structure of nicotine it is concluded that the pyrrolidine- $N$ is most probably the monofunctional group involved in sorption, the remaining part of the molecule being participative in the intermolecular attraction of the sorbed.

The Scatchard equation derived on the basis of a uniform site-binding model (binding of ligands to independent indistinguishable sites on the polymer) is (Weder et al., 1974)

$\frac{r}{C}=K n-K r$,

where $r, C, n$ and $K$ are defined above.

The Scatchard plot of an AAm/MA-nicotine system is shown in Fig. 6. The binding parameters of the Scatchard plot for the nicotine-AAm/MA hydrogel system were evaluated from the slope the intercept at the ordinate of the plateau at higher $r$ values. The derived values of the binding parameters $K$ and $n$ are listed in Table 2 for nicotine-AAm/MA hydrogel binding system.

On looking at the Scatchard plot in Fig. 6, it is seen that the plot is not linear downward as required by Eq. (8). Hence, the sorption process is not simple but a complicated one. The Scatchard plot is convex with a maximum. The convex plot indicates the operation of "mixed" cooperativity (both positive and negative cooperativity).

To determine the thermodynamic parameters of nicotine-hydrogel binding system, an adsorption experiment was made at $37^{\circ} \mathrm{C}$. Adsorption heat, adsorption free energy and adsorption entropy of this system were

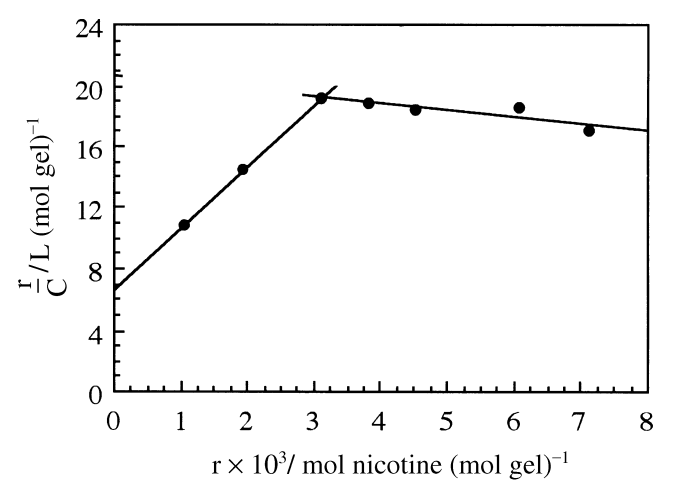

Fig. 6. Scatchard graph of nicotine - AAm/MA hydrogel binding system.

calculated using the following equations:

$\ln \frac{C_{2}}{C_{1}}=\frac{\Delta H}{R}\left(\frac{1}{T_{1}}-\frac{1}{T_{2}}\right)$

$\Delta G=-R T \ln K$,

$\Delta G=\Delta H-T \Delta S$,

where $C_{1}$ and $C_{2}$ are the free nicotine concentrations at the absolute temperature $T_{1}$ and $T_{2}$, respectively. $R$ is the universal gas constant; and $\Delta H, \Delta G$ and $\Delta S$ are heat of adsorption, free energy of adsorption, and entropy of adsorption, respectively.

The thermodynamic parameters of nicotine-hydrogel binding system are tabulated in Table 2.

In the nicotine-AAm/MA binding system, adsorption enthalpy and free energy of adsorption are found as negative value. So, this adsorption is an exothermic and spontaneous process. The adsorption of nicotine onto AAm/MA is a physical adsorption, because adsorption enthalpy is less than $20 \mathrm{~kJ} \mathrm{~mol}^{-1}$ (Table 2). As shown in Table 2, the sorption process is associated with negative enthalpy and positive entropy. Hence, both energetic and hydrophobic forces involve in the sorption process. Hydrogen bonding from the $\mathrm{N}$ atom is a likely candidate for the energetic forces. The intermolecular attraction between the nicotine molecules after sorption and the hydrophobic interaction, if any, between nicotine and AAm/MA hydrogel and the consequent changes in the water layer around the binding sites may be the contributing processes to the observed positive entropy change.

In the later experiments, the binding ratios of nicotine-hydrogel versus content of MA and, irradiation doses were plotted, as presented in Fig. 7. The binding ratios of AAm hydrogels increased after adding MA. But, the binding of nicotine is approximately constant with the increase of MA content in AAm/MA hydrogels. 
Table 2

Binding and thermodynamic parameters of nicotine-AAm/MA hydrogel system

\begin{tabular}{lllllll}
\hline$K_{i} / L \mathrm{~mol}^{-1}$ & $K / L \mathrm{~mol}^{-1}$ & $N$ & $u$ & $\Delta G / \mathrm{kJ} \mathrm{mol}^{-1}$ & $\Delta H / \mathrm{kJ} \mathrm{mol}^{-1}$ & $\Delta S / e u$ \\
\hline 20.64 & 448.82 & $45.97 \times 10^{-3}$ & 21.75 & -15.14 & 14.37 & 2.57 \\
\hline
\end{tabular}
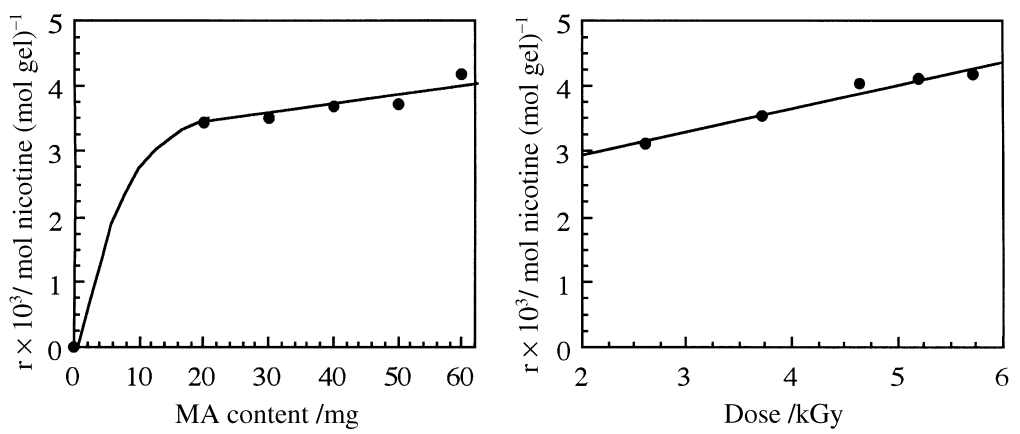

Fig. 7. The effect of MA content and irraditiation dose on the binding of nicotine onto AAm/MA hydrogel.

On the other hand, the binding ratio of nicotine slightly increased with increasing irradiation. Increasing of MA content and irradiation dose do not affect the binding of nicotine onto hydrogels, although the addition of MA to acrylamide does affect the binding of nicotine onto hydrogels.

\section{Conclusion}

Nicotine-selective poly(acrylamide/maleic acid) (AAm/MA) hydrogels were prepared by $\gamma$-irradiation. The swelling of AAm/MA hydrogel was increased in the following order;nicotine $>$ nicotinamide $>$ nikethami$\mathrm{de}>$ nicotinic acid $>$ water. Diffusions of water and the pharmaceuticals within the hydrogels were found to be non-Fickian in character.

Nicotine binding is thought to be one of the more important initial events which occurs upon the exposure of solid surfaces to the biological environment. AAm/ MA hydrogel sorbed only nicotine and did not sorb nicotinamide, nikethamide and nicotinic acid in the binding experiments. S-type adsorption in Giles's classification system was observed. The values of adsorption heat and free energy of this system were found to be negative whereas adsorption entropy was found to be positive. The adsorption of nicotine onto the hydrogel was found to be a physical adsorption.

Binding of nicotine into AAm hydrogels was found to increase with the addition of MA into the hydrogels. But this was approximately constant with increasing amount of MA in AAm/MA hydrogels. Adsorption of nicotine within AAm/MA hydrogels prepared with the same amount of MA gradually increased with the increase of irradiation doses. As a result, AAm/MA hydrogels can be used as an adsorbent for immobilization of pharmaceuticals such as nicotine.

\section{Acknowledgements}

The authors thank the members of the Polymer Group in the Department of Chemistry, Hacettepe University, for assisting in the preparation of hydrogels and also Dr. C. Uyan for providing pharmaceuticals.

\section{References}

Akkas,, P., Sari, M., sen, M., Güven, O., 1999. The effect of external stimuli on the Bovine Serum Albumin adsorption capacity of poly(acrylamide/maleic acid) hydrogels prepared by gamma rays. Radiat. Phys. Chem. 55, 717-721.

Giles, C.H., MacEwan, T.H., Nakhwa, S.N., Smith, D., 1960. Studies in adsorption. XI. A system of classification of solution adsorption isotherms, and its use in diagnosis of adsorption mechanisms and in measurement of specific surface areas of solids. J. Chem. Soc. 3973-3993.

Giles, C.H., D'Silva, A.P., Easton, I., 1974. A general treatment and classification of the solute adsorption isotherm. Part II. Experimental interpretation. J. Coll. Interface Sci. 47, 766-778.

Güven, O., sen, M., Karadağ, E., Saraydin, D., 1999. A review on the radiation synthesis of copolymeric hydrogels for adsorption and separation purposes. Radiat. Phys. Chem. 56, 381-386.

Kaetsu, I., 1996. Biomedical metarials, devices and drug delivery systems by radiation techniques. Radiat. Phys. Chem. 47, 419-423.

Karadağ, E., Saraydin, D., Öztop, H.N., Güven, O., 1994. Adsorption of bovine serum albumine to acrylamide-itaconic acid hydrogels. Polym. Adv. Technol. 5, 664-668. 
Karadağ, E., Saraydin, D., Güven, O., 1995. Behaviors of acrylamide/itaconic acid hydrogels in uptake of uranyl ions from aqueous solutions. Sep. Sci. Technol. 30, $3747-3760$.

Karadağ, E., Saraydin, D., Cetinkaya, S., Güven, O., 1996a. In vitro swelling studies and preliminary biocompatibility evaluation of acrylamide based hydrogels. Biomaterials 17, 67-70.

Karadağ, E., Saraydin, D., Güven, O., 1996b. Interaction of some cationic dyes with acrylamide/ itaconic acid hydrogels. J. Appl. Polym. Sci. 61, 2367-2372.

Karadağ, E., Saraydin, D., Güven, O., 1996c. A study on the adsorption of some cationic dyes onto acrylamide/itaconic acid hydrogels. Polym. Bullet. 36, 745-752.

Karadağ, E., Saraydin, D., Güven, O., 1997. Interaction of nicotine and its pharmaceutical derivatives with acrylamide/ itaconic acid hydrogels. J. Appl. Polym. Sci. 66, 733-739.

Kost, J., Langer, R., Gombotz, R., 1987.In: Peppas, N.A.(Ed.), Hydrogels in Medicine and Pharmacy, Vol. III. CRC Press, Florida.

Matsui, J., Kaneko, A., Miyoshi, Y., Yokoyama, K., Tamiya, E., Takeuchi, T., 1996. A molecularly imprinted nicotineselective polymer. Analyt. Lett. 29, 2071-2078.

Molyneux, P., 1984. Water-soluble Synthetic Polymers: Properties and Behavior, Vol. II. CRC Press, Florida.

Molyneux, P., Vekavakayanondha, S., 1986. The interaction of aromatic compounds with poly(vinylpyrrolidone) in aqueous solution. J. Chem. Soc. Faraday Trans. 1 (82), 291-317.

Peppas, N.A., Franson, N.M., 1983. The swelling interface number as criterion for prediction of diffusional solute release mechanisms in swellable polymers. J. Polym. Sci.: Polym. Phys. Ed. 21, 983-997.

Peniche, C., Cohen, M.A., Vasquez, B., Roman, J.S., 1997. Water sorption of flexible network based on 2-hydroxyethyl metachylate-triethylenglycol dimetacrylate copolymers. Polymer 38, 5977-5982.

Ritger, P.L., Peppas, N.A., 1987. Transport of penetrants in the macromolecular structure of coals 4 . Models for analysis of dynamic penetrant transport. Fuel 66, 815-826.

Rosiak, J., Burczak, K., Pekala, W., 1983. Polyacrylamide hydrogels as sustained release drug delivery dressing materials. Radiat. Phys. Chem. 22, 907-915.

Saraydin, D., Karadağ, E., Öztop, H.N., Güven, O., 1994. Adsorption of bovine serum albumin onto acrylamide/maleic acid hydrogels. Biomaterials 15, 917-920.

Saraydin, D., Karadağ, E., Cetinkaya, S., Güven, O., 1995a. Preparation of acrylamide/maleic acid hyrogels and their biocompatibility with some biochemical parameters of human sera. Radiat. Phys. Chem. 46, 1049-1052.

Saraydin, D., Karadağ, E., Güven, O., 1995b. Acrylamide/ maleic acid hydrogels. Polym. Adv. Technol. 6, 719-726.

Saraydin, D., Karadağ, E., Güven, O., 1995c. Adsorption of some heavy metal ions in aqueous solutions by acrylamide/ maleic acid hydrogels. Sep. Sci. Technol. 30, 3291-3302.

Saraydin, D., Karadağ, E., Güven, O., 1996a. Behaviors of acrylamide/maleic acid hydrogels in uptake of some cationic dyes from aqueous solutions. Sep. Sci. Technol. 31, 2359-2371.

Saraydin, D., Karadağ, E., Güven, O., 1996b. Adsorption of some basic dyes to acrylamide-maleic acid hydrogels. Sep. Sci. Technol. 31, 423-433.

Tümtürk, H., Çaykara, T., șen, M., Güven, O., 1999. Adsorption of $\alpha$-amilaz onto poly(acrylamide/maleic acid) hydrogels. Radiat. Phys. Chem. 55, 713-716.

Weder, H.G., Schildknecht, J., Lutz, R.A., Kessellring, P., 1974. Determination of binding parameters from Scatchard plots, theoretical and practical considerations. Eur. J. Biochem. 42, 475-481. 